\title{
Una introducción a la renta ciudadana en Italia
}

FABIO RAVELLI ${ }^{1}$

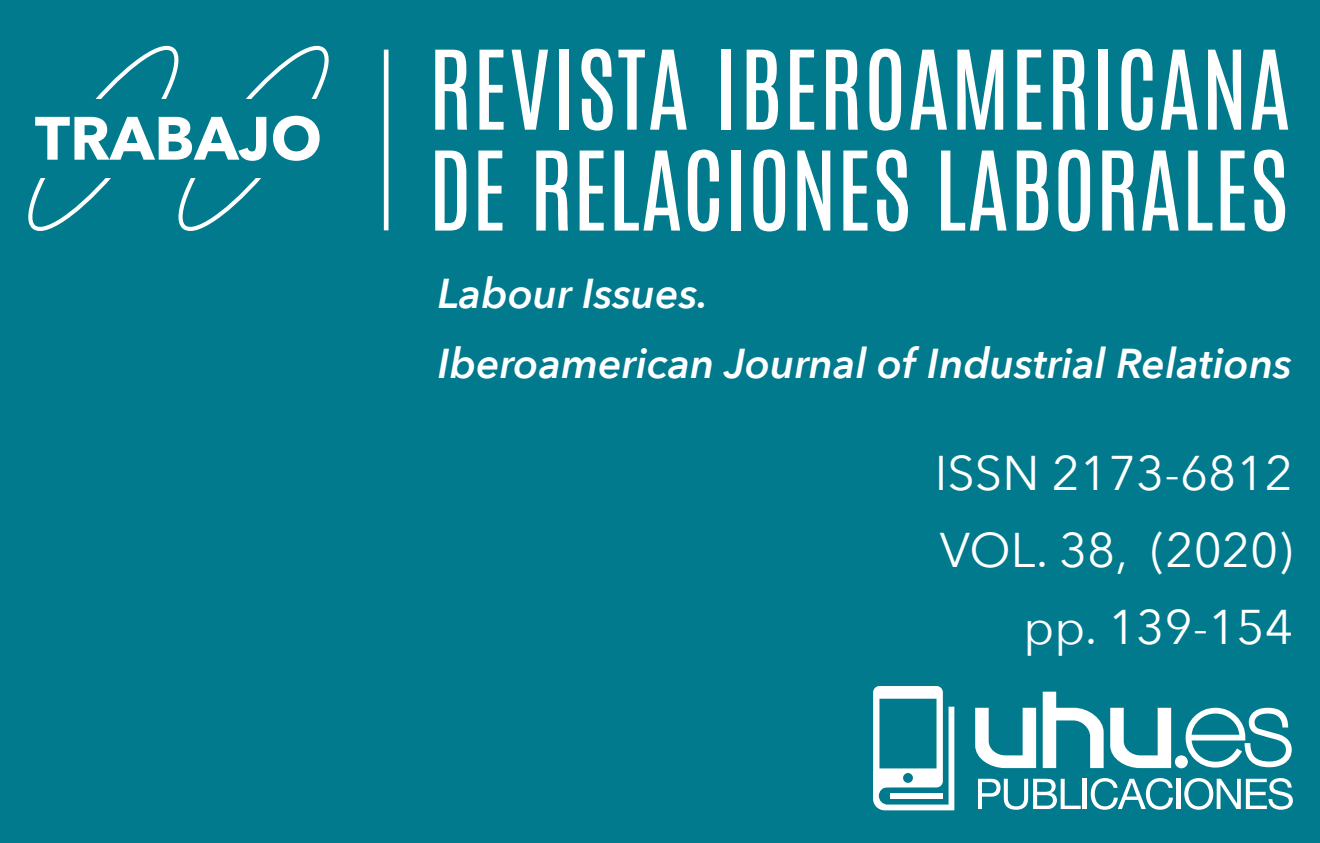




\section{FECHA RECEPCIÓN:}

2020-06-14

FECHA REVISIÓN:

2020-06-25

\section{Palabras clave}

La necesidad de implementar algún sistema relativo de garantía de la renta, como medida para fomentar la inclusión social y luchar contra el crecimiento progresivo de la población expuesta al riesgo de pobreza, ha constituido sigue construyendo una verdadera problemática también en el ámbito internacional. En este sentido y aunque de manera tardía, Italia ha conseguido también implementar entre sus herramientas normativas a nivel nacional una renta de inclusión a la que, con el tiempo, se rían añadiendo otros programas piloto, solo que esta vez, de orden regional. Algunos de estos programas no gozarian sin embargo de mucho éxito por la debilid sucho panteamiento y por cuestonestablilad cambios políticos, además de la falta de recursos económicos empleados para llevarlos a cabo. Por ello, el presente trabajo se plantea principalmente presentar el recorrido normativo experimentado por Italia descle la "Reforma Fornero" hasta la aprobación de la Ley del En hasta la aprobacion de la Ley del Empleo de 2014-2015, siendo trumentos la razón por la cuál surge, en 2017, la renta mínima de inserción y, más adelante, la renta ciudadana, profundizando a estos efectos en su conceptualización, funciones y desarrollo efectivo.

\section{RESUMEN:}

Renta ciudadana; inserción social; renta mínima de inserción; pobreza relativa.

\section{FECHA ACEPTACIÓN:}

2020-10-30

FECHA PUBLICACIÓN:

2020-12-05

Abstract

The need to implement some kind of income guarantee system, as a measure to promote social inclusion and fight the progressive growth of the population exposed to the risk of poverty, has been and continues to be a real problem also in the international field. In this sense, Italy has also succeeded, albeit belatedly, in implementing among its regulatory tools at the national level an income of inclusion to which, in time, other pilot programs would be added, but this time, at the regional level. Some of these programs would not be very successful, however, due to the weakness and instability of their approach and to issues of political change, in to carry them out. The present paper intends, mainly, to present the regulatory path experienced by Italy from the "Fornero Reform" to the approval of the 2014-2015 Employment Act, the inadequacy of these instruments being the reason why the minimum insertion income and leasen per pening for this purpose their conceptualization functions and effective development.

\section{KEYWORDS}

Citizen's income; social insertion; minimum insertion income; relative poverty.

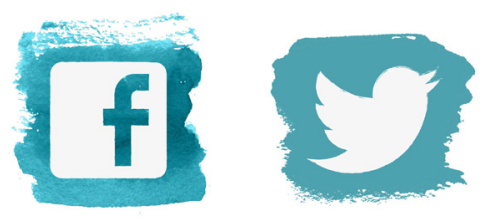




\section{UNA INTRODUCCIÓN A LA RENTA CIUDADANA EN ITALIA ${ }^{1}$}

\section{El retraso de Italia en la lucha contra la pobreza}

Desde hace unos años, en Italia se ha venido produciendo un crecimiento progresivo del número de personas expuestas al riesgo de pobreza. Los datos publicados por el Instituto Nacional de Estadística (ISTAT), correspondientes al año 2018, muestran un panorama preocupante: 5 millones de personas ( $8,4 \%$ de la población) se enfrentan a una situación de pobreza absoluta, mientras que más de 9 millones de personas (15\% de la población) se encuentran en una situación de pobreza relativa² ${ }^{2}$

Los instrumentos de lucha contra la pobreza establecidos en Italia han resultado se muy frágiles, por varias razones. La Comisión Europea observa que el marco de la política social "ha quedado fragmentado (excluida la atención de la salud), mostrando una capacidad de redistribución limitada, baja selectividad, baja calidad de los servicios, unas medidas de incentivación y activación limitadas y disparidades regionales importantes" ${ }^{3}$. En particular:

1. Proyecto RTI2018-098794-B-C32 denominado "Nuevas formas de protección social ante los cambios productivos" financiado por el MINECO/MICIU

2. ISTAT, La pobreza em Italia - 2018, en https://www.istat.it. La cesta de la pobreza absoluta representa "el conjunto de bienes y servicios que, en el contexto italiano, se consideran esenciales para que una familia determinada alcance un nivel de vida mínimamente aceptable.. Por otra parte, el umbral de pobreza relativa es igual "al gasto medio por persona en el país (es decir, es igual a gasto per cápita y se obtiene dividiendo el gasto del consumo total de los hogares entre el número de integrantes de dichos hogares)".

3. Véase Comisión Europea, Country Report. Italy 2016 including an in-depth review on the prevention and correction of macroeconomic imbalances, SWD(2016) 81 final, Bruselas, 26.2.2016 90 y ss./ Informe sobre Italia 2016, con un examen exhaustivo relativo a la prevención y la correc ción de los desequilbrios macroeconomicos, swD(2016) 81 final, Bruselas, 26.22016 , go y ss.
1) Durante mucho tiempo, Italia ha carecido de una medida general de lucha contra la pobreza, que se ha introducido por primera vez en 2017 con la renta de inclusión (en adelante Rel), regulada por el Decreto Legislativo No $147 / 2017^{4}$. Durante los últimos veinte años no ha faltado la experimentación ${ }^{5}$, pero el alcance de dicha medida se ha visto limitado, tanto en lo que respecta la duración de la misma, como en lo relativo a su ámbito de aplicación, por razones atribuibles a la falta de un marco normativo general y a la falta de recursos asignados

2) La inercia del Estado ha puesto sobre los hombros de las Regiones la responsabilidad de tomar una iniciativa legislativa en el ámbito de la lucha contra la pobreza. Algunas Regiones han tratado de introducir planes de ingresos mínimos que pudiesen ofrecer protección al menos a las personas más marginadas socialmente ${ }^{6}$. Sin embargo, la acción de las Regiones ha resultado débil y se ha caracterizado por una cierta inestabilidad de los programas a lo largo del tiempo. El Ingreso Mínimo Regional surgió en todo el territorio nacional como un experimento y, en la mayoría de los casos, no llegó a superar el período de prueba, tanto por razones vinculadas a la falta de recursos disponibles como por razones vinculadas a los cambios políticos en los consejos regionales.

La falta de continuidad en el tiempo ha tenido dos consecuencias: en primer lugar, ha hecho que en muchos casos las políticas regionales de lucha contra la pobreza sean poco realistas; en segundo lugar, ha dificultado especialmente la evaluación de los efectos que esas medidas han provocado en el territorio y, en última instancia, su utilidad (con vistas, posiblemente, a su recalibración);

3) Operativamente, la gestión de los servicios sociales para la lucha contra la pobreza incumbe, principalmente, a las autoridades locales (municipios), que, sin embargo, a lo

4. Para más información sobre los ingresos por inclusión, véase Alaimo 2017; Vincieri 2017; Ravelli 2018a. El hecho de no prever una medida general contra la pobreza representaba un claro incumplimiento de la disposición constitucional que obliga al Estado a "determinar los niveles esenciales de las prestaciones relativas a los derechos civiles y sociales que deben garantizarse en todo el territorio nacional" (art. 117, párr. 2, letra m), Cost)

5. Pensemos, por ejemplo, en las siguientes medidas: la "renta mínima de inserción" (RMI) introducida por el gobierno de Prodi en 1998 y derogada en 2001; la "tarjeta social", introducida en 2008 por el gobierno de Berlusconi; el "apoyo a la inclusión activa" (SIA), introducido experimenalmente en 2012 por el gobierno de Monti y renovado por gobiernos posteriores, hasta que quedó reemplazado definitivamente en 2017 por la Rel.

6 . Véanse, entre los ejemplos más significativos, los planes de ingresos mínimos introducidos por Lazio,

Puglia, Campania, Basilicata, Friuli Venezia Giulia. Para una visión general de las principales experiencias regionales, véase Toso 2016, pág. 112 y ss. y Granaglia, Bolzoni 2016, pág. 95 y ss. 
largo de los años han ido reduciendo gradualmente la transferencia de recursos generados por el Estado;

4) Por último, las dificultades con las que se ha ido tropezando el sistema italiano en la lucha contra la pobreza se deben, generalmente, al enfoque categórico de los seguros sociales, encuadrados dentro del sistema de seguridad social italiano, que ha provocado un desequilibrio en la composición del gasto social, ya que una parte muy importante de los recursos es absorbida por las transferencias monetarias destinadas a financiar las pensiones ${ }^{7}$.

La idea básica que inspiró durante mucho tiempo al legislador nacional - y que se en cuentra presente en los artículos 4 y 38 de la Constitución italiana - fue que la principa barrera anti pobreza tenía que ser el trabajo, al que todos (en teoría) debían tener acceso, puesto que se suponía que el trabajo era la clave para la inclusión social ${ }^{8}$. En caso de que el trabajador perdiera la posibilidad de trabajar, debido a la ocurrencia de acontecimientos relacionados con ciertos riesgos sociales ${ }^{9}$, se beneficiaría de los instrumentos de protección ofrecidos por el sistema de seguridad social, siguiendo el modelo basado en la idea del "seguro social" bismarckiano ( art. 38. 2, Cost.) ${ }^{10}$

Los "inaptos para el trabajo", en la medida en que estaban excluidos de la posibilidad de generar por sí mismos ingresos en concepto de contrapartida por el desempeño de una actividad laboral, tenían derecho a la manutención y a la asistencia social (véase e artículo 38.1, Const.): por lo tanto, en la mente de los constituyentes, la asistencia debiera tener una función meramente residual. Sin embargo, el mecanismo que acabamos de describir, aparentemente bien pensado, al poco tiempo se vería "atascado".

Resulta evidente que el objetivo del pleno empleo, implícitamente recogido en la afirmación "derecho al trabajo" a la que se refiere el artículo 4.1 de la Constitución, no pudo

7. Reyneri 2015, págs. 100 a 102. Véase también Istat, Informe Anual 2016, texto disponible en: http://www.istat.it.

8. La literatura sobre el papel del trabajo en el sistema constitucional italiano es muy extensa. Sin pretender ser exhaustivos, entre las más recientes contribuciones ofrecidas por los abogados aboralistas podemos señalar: Loy 2009; Nogler 2009; Pedrazzoli 2011. Véase también la contribución más reciente, pero aún fundamental, de Mancini 1975.

9. El 38.2 de la Constitución, en el que se exponen las características fundamentales del sistema de seguridad social italiano, se refiere a "accidente, enfermedad, invalidez, vejez, desempleo

involuntario", a lo que habría que añadir la maternidad, aunque no aparezca expresamente en la disposición constitucional.

10. Sobre el concepto de "seguridad social", véase Balandi 1987. ser conseguido ${ }^{11}$. Un considerable número de personas terminó encontrándose ante una situación de falta de empleo, pero sólo una parte de todas aquellas personas llegó a beneficiarse de la protección que ofrecía el sistema de seguridad social, a través de un seguro destinado a proteger a los trabajadores (y que ni siquiera llegaba a cubrirlos a todos) frente al riesgo de la pérdida del empleo.

En primer lugar, los trabajadores autónomos estaban excluidos del ámbito de la protección por desempleo: $y$, dentro del colectivo de trabajadores asalariados, también quedaban excluidos de la protección por desempleo los gerentes ${ }^{12}$ y los trabajadores del sector público.

Por otra parte, se encontraban ante la dificultad de acceder a las prestaciones por desempleo, las personas "subempleadas" (que trabajaban en empleos precarios), es decir, aquellas que, mientras estaban trabajando, no cumplían los requisitos mínimos para acceder a tal prestación debido a la inseguridad laboral extrema a la que estaban expuestos. También se encontraban desprotegidos los desempleados de larga duración que habían agotado el período máximo del derecho a la prestaciones (para el NASPI, 24 meses). Por último, los llamados "desocupados", es decir, los que nunca habían podido entrar a mercado laboral y que, por lo tanto, no tenían la condición de "trabajador" necesaria para beneficiarse de las medidas contra el desempleo involuntario, permanecían al margen del sistema protector: de hecho, al no ser calificados como "no aptos para el trabajo", no podían disfrutar de la protección social ${ }^{13}$.

Por lo tanto, los dos métodos imaginados por el legislador constitucional para combatir los fenómenos de la pobreza y la marginación social no han resultado eficaces.

11. Las razones del fracaso del objetivo de conseguir pleno empleo son complejas y sólo pueden mencionarse brevemente. En resumen, puede decirse que, si bien algunas de estas razones afectan a todos los sistemas económicos occidentales, otras se refieren a perfiles específicos de fragilidad del sistema italiano. El cambio de paradigma inducido por la tecnología, que genera fenómenos de crecimiento sin empleo, y las crisis económicas que afectan cíclicamente a los mercados laborales europeos son razones inherentes al contexto general común a todos los países miembros de la UE. Por otra parte, se pueden calificar como características peculiares del sistema italiano: la baja competitividad del sistema productivo; el retraso en el desarrollo del sector terciario (y en particular del sector de los servicios sociales); la pronunciada segmentación del mercado laboral; la baja calificación de la estructura del empleo; los bajos niveles de participación de las mujeres y los trabajadores de edad. Sobre esta idea, ver Fellini 2015

12. Sin embargo, los trabajadores para subordinados (es decir, falsos autónomos), pueden beneficiarse del llamado DIS-COLL, una forma de subsidio por desempleo, equiparado al previsto para los trabajadores asalariados (el llamado NASPI). Tanto el NASPI como el DIS-COLL se rigen por el Decreto Legislativo n 22/2015.

3. Para más información al respecto, véase Bozzao 2018. 
Por una parte, el fracaso del objetivo de alcanzar el pleno empleo, demuestra que e trabajo no está al alcance de todos aquellos que lo desean. Además, incluso cuando resulte accesible, el trabajo ya no puede considerarse, en sentido absoluto, el mejor seguro contra la pobreza, como lo demuestra sobradamente la existencia de la denominada categoría de "trabajadores pobres"14. Por otra parte, el sistema de seguridad social, basado en la idea de la solidaridad categórica, y fuertemente gravado por el principio asegurador, no ha sido capaz de interceptar todas las situaciones de necesidad presentes en la sociedad, dejando a los "nuevos pobres" sin protección ${ }^{15}$.

Había dos posibles maneras de remediar esta situación y el legislador italiano, en diferentes momentos, se ha decantado por ambas. Una primera solución consistió en ampliar en la medida de lo posible, o bien, en la medida en que lo permitiera la naturaleza de instrumento, el grado de cobertura que ofrecían los planes de seguros sociales. Ello se llevó a cabo con la aprobación de la llamada "Reforma Fornero" (2012) ${ }^{16}$ y con los decretos que regulaban la llamada "Ley de Empleo II" (2014-2015)17, pero aún así resultó ser una solución insuficiente.

Observando la insuficiencia de los instrumentos de protección tradicionalmente utilizados en 2017 el legislador decidió finalmente seguir un segundo camino, que condujo a la introducción de una medida general contra la pobreza, la "renta mínima de inserción" (RMI), regulada por el Decreto Legislativo $N^{0} 147 / 20171^{18}$. La medida fue de corta duración, ya que se vio sustituida, en los primeros meses del año 2019, por la nueva "renta de la ciudadanía/renta ciudadana” (RdC) prevista en la Ley $n^{\circ} 26 / 2019$, que convirtió en Ley el Decreto n $4 / 2019^{19}$

14. Según datos de Eurostat, en 2017 la tasa de pobreza de los trabajadores en Italia era de $12,3 \%$, frente a una media europea del 9,6\%: véase Peña-Casasas, Ghailani, Spasova, Vanhercke 2019. Para un análisis de la situación italiana, véase Raitano, Jessoula, Natili, Pavolini 2019

15. Bozzao 2018. Per un approfondimento sulla povertà in Italia cfr. Saraceno 2015.

16. Ley n. $92 / 2012$

17. Véanse en particular los Decretos legislativos № 22/2015, 148/2015 y 150/2015, de aplicación de la ley delegada № 183/2014.

18. El artículo 2 del Decreto Legislativo № 147/2017 define la Rel como "una medida de carácter universal, condicionada a la prueba de medios y a la adhesión a un proyecto personalizado de activación e inclusión social y laboral, destinado a liberar a las personas de la pobreza”. La Rel es un medida dirigida a las familias, y no a los individuos: es de naturaleza compuesta, ya que se compone de un apoyo monetario (un subsidio clásico) y de un conjunto de servicios destinados a favorecer la recuperación del rumbo natural de una familia, a la luz de una evaluación multidimensional de su necesidad, en una condición de pobreza, siempre que se adhiera a un proyecto personalizado espeć́fico, adaptado a la familia beneficiaria en base de las necesidades de sus miembros.

19. Para un primer comentario sobre la disciplina de la RdC, véanse los comentarios más amplios en Sigillò Massara 2019 y Giubboni 2020; véase también Casillo 2019, D’Onghia 2020 y Vincieri 2020.

\section{Concepto y funciones de la renta ciudadana.}

Antes de profundizar en el análisis de los rasgos esenciales de esta nueva disciplina, resulta oportuno aclarar el significado de la expresión "renta ciudadana". En el debate político sobre la renta mínima, estas palabras suelen hacer referencia a un beneficio de carácter universal e incondicional que una comunidad política proporciona a todos sus miembros, independientemente de toda consideración de la situación subjetiva del beneficiario, según el modelo de renta básica preconizado por Philippe Van Parijs ${ }^{20}$.

En realidad, la renta ciudadana italiana - llamada así por la elección deliberada de utilizar una fórmula más atractiva y electoralmente remunerativa - debe ser atribuida a la diferente tipología de renta mínima selectiva, doblemente condicionada a la evaluación de estado de necesidad y a la activación del beneficiario, situándose así en el cauce trazado por la Rel, de la que es heredera directa ${ }^{21}$.

Al igual que la Rel, la RdC muestra un sólido anclaje en el modelo europeo de ingresos mínimos, del que hereda las características básicas resumidas en el principio $n^{\circ} 14$ de llamado Pilar Social: "Toda persona que carezca de recursos suficientes tiene derecho a unas prestaciones de renta mínima adecuadas que garanticen una vida digna a lo largo de todas las etapas de la vida, así como el acceso a bienes y servicios de capacitación.".

Para los que pueden trabajar, el ingreso mínimo debería combinarse con incentivos para la (re)integración en el mercado laboral ${ }^{22}$

En comparación con la Rel, definida como una "medida nacional única para combatir la pobreza y la exclusión social" ( artículo 2 del Decreto Legislativo 147/2017), la RdC parece que se propone desempeñar unas funciones más amplias. De hecho, el artículo 1 del Decreto Legislativo № 4/2019 la describe como "una medida fundamental de la política laboral activa para garantizar el derecho al trabajo, luchar contra la pobreza, la desigualdad y la exclusión social y promover el derecho a la información, la educación, la formación y la cultura mediante políticas destinadas a apoyar económicamente y a integrar socialmente en la sociedad y en el mundo laboral a las personas que se encuentren en riesgo de exclusión ".

La comparación textual de las dos medidas muestra, además de una mejora evidente en la RdC en el plano funcional, un "desplazamiento" del enfoque de este medida desde

20. Van Parijs 1991.

21. Para una clasificación de los diversos tipos de ingresos mínimos, véase Toso 2016 y Ravelli 2018a.

22. Comisión Europea El Pilar Europeo de Derechos Sociales, Oficina de Publicaciones de la Unión Europea, Bruselas, 2017. 
la lógica de la asistencia a las personas expuestas al riesgo de pobreza, hacia la de su activación, en vista a su (re)incorporación en el mercado laboral ${ }^{23}$.

El orden en el que se enumeran enumerados los objetivos perseguidos por la medida en cuestión no es accidental: la garantía del derecho al trabajo parece constituir, según las intenciones del legislador, un prius lógico con respecto a la lucha contra la pobreza. Además, este enfoque del problema crea claramente un efecto eco de lo recomendado por la resolución del Parlamento Europeo de 24 de octubre de 2017, la cual, en la idea de sugerir un enfoque estratégico integrado que tenga en cuenta el carácter multidimensiona de la pobreza, afirma que "el objetivo real de los regímenes de renta mínima no debe ser la mera asistencia, sino sobre todo acompañar a los beneficiarios en su paso de situaciones de exclusión social a la vida activa, evitando así la dependencia a largo plazo"24

Sin duda, hay algo de verdad en la afirmación conforme a la cual: lo que el legislador quiso fue "destacar el espíritu laboral de la nueva ayuda"25.

Sin embargo, más allá de la evidente diferencia en términos de definición, así como de la existencia, en la nueva medida, de algunos elementos que nos lleven a pensar en una adhesión más marcada respecto al primer modelo de política, la relación entre la Rel y la RdC está más fundamentada en la continuidad que en la discontinuidad ${ }^{26}$

En cuanto al enfoque general, no existe una diferencia tan dramática entre las dos medidas, ya que los puntos de contacto superan con creces los de divergencia: ambas medidas son selectivas, condicionales y compuestas, es decir, consisten en una prestación (a la que se accede en función de determinados índices de pobreza) y en un conjunto de servicios destinados principalmente a la integración laboral y, en casos más complejos, a la inclusión social. Un discurso aparte sería la denominada "pensión de ciudadanía" o pensión ciudadana (PdC), es decir, la configuración que asume la RdC en los casos en que la prestación tiene por objeto apoyar a "las familias compuestos exclusivamente por uno o más miembros con 67 años de edad o más". (artículo 1.2). La pensión ciudadana, que es una de las principales novedades introducidas por la Ley № $26 / 2019$, persigue objetivos parcialmente diferentes de los que caracterizan a la RdC: al ser una medida dirigida a quienes, por su edad, están excluidos de la población activa, se califica exclusivamente como un instrumento de lucha contra la pobreza. La pensión de ciudadanía rompe, por tanto, el vínculo entre subsidio y activación que es propio del RdC, cosa que determina su carácter híbrido como medida de lucha contra la

23. Alaimo 2019

24. Resolución del Parlamento Europeo, de 24 de octubre de 2017, sobre las políticas encaminadas a garantizar la renta mínima como instrumento para luchar contra la pobreza, punto 25.

25. Alaimo 2019

26. Baldini, Gori 2019. pobreza y como instrumento de política activa. Como la idea de inserción de los beneficiarios en el mercado laboral ya no sería un objetivo en este caso, la pensión de ciudadanía entra naturalmente en el ámbito de las medidas puramente asistenciales ${ }^{27}$

\section{Los beneficiarios.}

En lo que respecta a la identificación de los beneficiarios, el ingreso mínimo puede adoptar, en abstracto, tanto la forma de una medida individual como la forma de una medida de apoyo familiar. A este propósito, puede resultar útil examinar el marco normativo europeo que, en la Recomendación 92/441/CEE, deja abiertas ambas vías, en la medida en que recomienda "conceder a las personas cuyos recursos, calculados por individuo o por familia, sean inferiores a los importes así fijados, adaptados o completados, una ayuda financiera diferencial que les permita disponer de dichos importes; " (véase el punto C) 2 de la Recomendación 92/441/CEE) ${ }^{28}$

Partiendo del análisis de la experiencia de otros países europeos, que constituye un punto de referencia obligatorio, el derecho a la prestación se concederá generalmente a individuo y no al hogar o núcleo familiar ${ }^{29}$. Esta solución, pues, parece ser más ajustada a lo dispuesto en el artículo 34 de la Carta de lo Derechos Fundamentales de la UE, en el que la garantía de una existencia digna a todos aquellos que no dispongan de recursos suficientes, se configura como un derecho individual ${ }^{30}$.

El legislador italiano, sin embargo, prefirió introducir el ingreso mínimo como una

medida de carácter familiar. Ello no debería sorprender ya que le encontramos una explicación: por un lado, en la particular "prudencia" (aunque podrían ser términos más adecuados la "desconfianza" y "reticencia") con la que el legislador italiano abordó la cuestión de los ingresos mínimos y, por otro lado, en razones de coherencia sistemática puesto que otras prestaciones de carácter asistencial vigentes en el ordenamiento jurídico italiano se conceden con la condición de que se demuestre la situación de pobreza de la unidad familiar de origen del solicitante. El acceso a la RdC conlleva, obviamente, el cumplimiento conjunto de una serie de requisitos (véase el artículo 2 ).

27. Sobre la pensión de ciudadanía véase Casillo 2019 pp. 572-573.

28. Recomendación del Consejo, de 24 de junio de 1992, sobre los criterios comunes relativos a recursos y prestaciones suficientes en los sistemas de protección social, 92/441/CEE, Diario Oficial $n^{\circ} \mathrm{L} 245$ de 26/08/1992

29. Ravelli 2018a, pp. 102-103

30. Bronzini 2011, p. 230; in senso adesivo, Vincieri 2020. 
A) Un primer grupo de requisitos se refiere a la situación económica y patrimonial de la

unidad familiar beneficiaria de la ayuda: en particular, la unidad familiar debe tener: un valor ISEE inferior ${ }^{31}$ a 9.360 euros; un valor del patrimonio inmobiliario, ex cluida la vivienda, no superior a 30.000 euros; un valor del patrimonio mobiliario no superior a 6.000 euros; un valor de la renta familiar inferior al umbral de 6.000 euros anuales (o 9.360 euros en caso de que la unidad familiar resida en una vivienda de alquiler ${ }^{32}$. Además, la unidad familiar no deberá disponer de ningún tipo de bienes duraderos (por ejemplo, vehículos o barcos recientemente registrados) B) Más delicado resulta el tema relacionado con el cumplimiento del requisito de ciudadanía o residencia. La persona que tiene la intención de solicitar la $\mathrm{RdC}$, deberá se ciudadano italiano o ciudadano europeo (o familiar del mismo); también puede tratarse de un nacional proveniente de un tercer país, en cuyo caso deberá estar en posesión de un permiso de residencia en la UE de larga duración. ${ }^{33}$

Por consiguiente, los extranjeros que estén en posesión de un permiso de residencia válido, pero que no tengan la consideración de residentes de larga duración, quedarán excluidos del acceso a la Renta Ciudadana. Además, el legislador del año 2019 no realizó ningún cambio al respecto, limitándose a confirmar el planteamiento adoptado por e Decreto Legislativo n 147/2017 sobre el acceso a la Renta de Inserción. La elección de legislador italiano resulta cuestionable, tanto desde el punto de vista del derecho comunitario como del derecho interno.

Desde la perspectiva del derecho europeo, parece objetable el hecho de excluir de la posibilidad de disfrute de la Renta Ciudadana a los titulares del denominado "permiso de trabajo único" al que se refiere la Directiva 2011/98/UE, cuyo artículo 12 recoje el derecho de los nacionales de terceros países a la igualdad de trato en lo que respecta a "los ámbitos de la seguridad social definidos en el Reglamento (CE) N 883/2004" (véase el artículo 12.1 e) de la Directiva). La RdC, que estructuralmente tiene connotaciones de "prestación familiar" en el artículo 3.1 j) del Reglamento, podría considerarse incluida entre las "pres-

31. EI ISEE ("Indicador de la Situación Económica Equivalente") es el indicador que se utiliza para evaluar la situación económica de los hogares que desean solicitar una prestación social subvencionada: para cumplir con la "prueba de medios" necesaria para acceder a las prestaciones de asistencia social, el valor del ISEE debe ser inferior al umbral establecido por el legislador en cada caso. Para beneficiarse de la RdC, el núcleo familiar debe asimismo demostrar un valor ISEE bajo.

32. Este valor de la renta tiene en cuenta un hogar formado por un solo individuo. Si el hogar está formado por varios individuos, la cifra se multiplica por una escala de equivalencia.

33. La ampliación de la RdC a los ciudadanos europeos (y a sus familiares) y a los nacionales de terceros países (siempre que sean residentes de larga duración) aplica los requisitos de las Directivas 2004/38/CE y 2003/109/CE, respectivamente. taciones especiales en metálico de carácter no contributivo" a las que alude el artículo 70.2 y, por tanto, entraría dentro del ámbito de aplicación de la Directiva 2011/98/UE ${ }^{34}$

Otra exclusión de dudosa legitimidad es la que afecta a los beneficiarios de protección internacional, que tendrán derecho a recibir, "en el Estado miembro que les haya concedido tal protección, la asistencia social necesaria, en las mismas condiciones que los nacionales de dicho Estado miembro. " (artículo 29.1 de la Directiva 2011/95/UE). Desde este punto de vista, a fin de evitar incumplimientos de la legislación europea, el artículo 2 del decreto que regula la RdC debería ser completado, de tal forma que incluya a los refugiados y a las personas que gozan de protección subsidiaria entre los beneficiarios de dicha medida.

La exclusión de la posibilidad de disfrute de la RdC de los nacionales provenientes de terceros países, también suscita muchas preocupaciones desde el punto de vista del derecho constitucional. La jurisprudencia Constitucional ha ido completando de manera progresiva un proceso de transformaciones del sistema de seguridad social italiano en un sentido más inclusivo, promoviendo la aplicación del principio de solidaridad de tipo "cosmopolita" y determinando la superación sustancial de la distinción entre ciudadano y extranjero en lo que respecta el disfrute de los derechos sociales ${ }^{35}$, particularmente cuando se trata de acceder a medidas destinadas a "permitir la satisfacción concreta de las necesidades primarias inherentes a la misma esfera de protección del ser humano"; En otras palabras, ha quedado expresado que la existencia de un "núcleo" de prestaciones esenciales constituye, en palabras de los tribunales, "un parámetro de igualdad de trato inevitable entre los ciudadanos y los extranjeros que residen legalmente en el territorio del Estado" (Sentencia del Tribunal Constitucional № 187/2010). En su posterior fallo $N^{\circ}$ 329/2011, el TC especificó que la necesidad de garantizar a los nacionales de terceros países el acceso a las medidas destinadas a satisfacer las "necesidades básicas" se ajusta "a los principios de solidaridad social primordial, asumidos como valor fundador de los mismos derechos inalienables de la persona, que no permiten ningún tipo de distinción en función de cualquier tipo de calidad o posición subjetiva y, por tanto, tampoco permite trato diferente a causa de la distinta condición de ciudadano o extranjero"36.

34. La jurisprudencia del Tribunal de Justicia sugiere que esta reconstrucción es plausible. En el caso CJEU, de 21 de junio de 2017, Caso C-449/16, Del Rosario Martínez Silva, el Tribunal sostuvo que una prestación familiar no contributiva -el subsidio para familias numerosas- no debe considerarse una prestación asistencial sino una prestación de "seguridad social" a tenor del Reglamento 883/2004; por consiguiente, en lo que respecta al acceso a dicha prestación, se aplica el principio de igualdad de trato establecido por la Directiva 2001/98/UE. Sobre esta idea, consultar: Ravelli 2018a, p. 169; en el mismo sentido, ver Rosafalco 2019, p. 89, y Vincieri 2020. Contra Casillo 2019, p. 567. 35. Apostoli 2016.

36. II medesimo argomento è stato sviluppato anche nelle sentenze n. 40/2013, n. 222/2013, n $22 / 2015$ e n. $230 / 2015$ 
C) Por último, la persona que solicita la RdC tendrá que haber residido en Italia du rante al menos 10 años, de los cuales los dos últimos de forma ininterrumpida. En este supuesto concreto y en comparación con el pasado reciente, existe un endurecimiento significativo de este requisito, ya que el Decreto Legislativo № 147/2017 solo se limitaba a exigir la residencia continuada durante dos años. Si bien el requisito de los dos años de residencia no planteó problemas particulares, ya que ese período de tiempo no constituye una discriminación indirecta, el hecho de elevar dicho período a diez años plantea más de una preocupación: en particular, es dudoso que el requisito de diez años de residencia, cuando da lugar a una desventaja para los extranjeros, pueda considerarse compatible con el principio de igualdad de trato establecido por las Directivas 2003/109 y $2011 / 98^{37}$

Además, en términos de legitimidad constitucional, un período mínimo de diez años podría considerarse excesivamente largo, aunque las señales enviadas por la Consulta en los últimos años sobre cuestiones similares, no son inequívocas. Por un lado, el requisito de residencia de diez años para acceder al subsidio social previsto en la Ley 335/1995 se ha considerado legítimo en más de una ocasión (la última vez, en marzo de 2019) ${ }^{38}$, lo que sugiere que un posible juicio de constitucionalidad que tuviera un requisito de residencia similar para acceder a la RdC, se llevaría a cabo utilizando el mismo criterio de evaluación. Sin embargo, el Tribunal Constitucional, en 2018, dictó un conjunto de sentencias en las que concretaba la ilegalidad -sub specie de irrazonabilidad- de los requisitos de residencia expresados en varios años que establecían algunas leyes regionales sobre e acceso a la asistencia social ${ }^{39}$

Por lo tanto, la cuestión está lejos de ser pacífica: por el momento, sin embargo, no está claro si la Corte quiere mantener una doble vía de evaluación - distinguiendo entre e bienestar regional y el bienestar estatal - o si, por el contrario, la diversidad de soluciones adoptadas es indicativa de un conflicto de directrices destinadas a ser recompuestas de cara al futuro.

\section{La naturaleza compuesta de la medida: a) el subsidio:}

La prestación ofrecida por la RdC tiene una naturaleza compuesta. Implica el uso tanto de la política pasiva más tradicional (el subsidio) como de la política activa (servicios de reinserción laboral e inclusión social). La solución adoptada por el legislador italiano no

37. Casillo 2019, p. 567; Ravelli 2019, p. 184.

38. STC n. 50/2019.

39. SSTC n. 106/2018, n. 107/2018 e n. 166/2018. difiere de las que se practican habitualmente en el resto de Europa y se ajusta a lo recomendado por el Parlamento Europeo en la mencionada Resolución de 24/10/201740.

De conformidad con el artículo 3 de la misma, el beneficio económico consiste en dos elementos ${ }^{41}$ :

- 1) un componente para complementar los ingresos familiares, hasta el umbral de 6 000 euros por año ${ }^{42}$, multiplicado por el parámetro correspondiente de la escala de equivalencia a efectos de la $\mathrm{RdC}^{43}$;

- 2) un componente para complementar los ingresos de las familias residentes en viviendas alquiladas, en una cantidad igual al importe de la renta anual prevista en el contrato de alquiler, según lo declarado a efectos de la ISEE, hasta un máximo de 3.360 euros anuales.

40. Resolución del Parlamento Europeo de 24/10/2017, citada anteriormente, en la que se afirma que "la renta mínima debe ser la última red de protección social y consistir en un apoyo financiero adecuado complementario a un acceso garantizado a servicios de calidad y a políticas activas de empleo, como el medio más eficaz para luchar contra la pobreza y garantizar una existencia digna a todos aquellos que no disponen de recursos suficientes;" ( punto 1). Se especifica además que "los regímenes de renta mínima deben enmarcarse en un enfoque estratégico dirigido a la integración y la inclusión social, que comprenda políticas generales y medidas específicas relativas a la vivienda, la asistencia sanitaria, la educación y formación, los servicios sociales y tros servicios de interés general, para ayudar a las personas a superar la pobreza, aportando un apoyo personalizado y ayuda para lograr acceder al mercado de trabajo a las personas capaces de trabajar;" ( el punto 25).

41 El subsidio se proporciona mediante la llamada "Tarjeta RdC", una tarjeta electrónica recargable que puede utilizarse para adquirir bienes y servicios, así como para realizar retiros de efectivo (dentro de los límites establecidos)

42. El umbral se eleva a 7560 euros si el ingreso mínimo toma la forma de una "pensión de ciudadanía"; La soglia è aumentata a euro 7560 nel caso in cui il reddito minimo assuma la configurazione di "pensione di cittadinanza".

43. Por ejemplo, supongamos un ingreso anual de 4530 euros. Si la familia está compuesta por un solo miembro, la RdC complementará los ingresos hasta un máximo de 6.000 euros (6.000$4530=1470$, o 122,50 euros por mes). Si estos ingresos serían atribuibles a una familia compuesta por dos adultos y un menor, el umbral máximo de 6.000 debe multiplicarse por el parámetro correspondiente de la escala de

equivalencia, que tiene en cuenta el tamaño de la unidad familiar: por lo tanto, $(6.000 \times 1,6)$ $4530=5.070$, lo que equivale a 422,50 euros al mes. Si la familia de referencia está compuesta por tres adultos y un menor, la aplicación de un parámetro de equivalencia de 2,1 daría lugar, con unos ingresos iniciales de 4.530 euros, a un suplemento de 672,50 euros al mes 
Por lo tanto, un núcleo familiar compuesto por una sola persona podría beneficiarse de una ayuda máxima de 9.360 euros al año, o 780 euros al mes: esta cantidad no es aleatoria, sino que tiene en cuenta las recomendaciones de la Resolución del Parlamento Europeo de 20/10/2010, en la que se consigna que " los regímenes de renta mínima adecuados deben establecerse en un nivel de al menos el $60 \%$ de la mediana de la renta en el Estado miembro de que se trate" 44 . Por consiguiente, el legislador de 2019 ha tratado de darle un remedio a uno de los principales puntos críticos de la Rel, a sabiendas de la insuficiencia de financiación de la medida, según admite el propio Gobierno, para cubri la mayoría de las situaciones de pobreza ${ }^{45}$. Aunque el esfuerzo es digno de aprecio, aún subsisten algunas preocupaciones sobre la definición de la escala de equivalencia, ya que la cuantía del subsidio parece ser más ventajosa para las personas solteras que para las familias numerosas, aunque son estas últimas las que tienen que experimentar las condiciones más duras de la pobreza absoluta ${ }^{46}$. Por lo tanto, resultaría apropiado revisar dicha escala de equivalencia.

El subsidio se concede siempre que se cumplan los requisitos de acceso, hasta una duración máxima de 18 meses. Si las condiciones de necesidad persisten más allá de este período, la Renta Ciudadana puede ser renovada tras un período de suspensión de un mes. La suspensión no operará en el caso del pago de la pensión ciudadana.

Una importante novedad que trae la RdC, a diferencia de la Rel, se refiere a la posibilidad de acumular el subsidio, dentro de ciertos límites, con otras prestaciones sociales, en particular, con el subsidio por desempleo. En el párrafo 8 del artículo 2 se establece que "la Renta Ciudadana es compatible con el disfrute de la Nueva Prestación de la Seguri- dad Social para el Empleo (NASPI según su abreviatura en italiano) y la con prestación por desempleo para los trabajadores con una relación de cooperación coordinada (DISCOLL) [...] y con otro instrumento de apoyo a los ingresos en caso de desempleo involuntario". Se trata, por tanto, de una modificación benéfica.

En realidad, no es difícil imaginar el caso de una familia numerosa, compuesta por dos adultos y tres niños, en la que sólo uno de los miembros recibe el subsidio por desempleo. En tal caso, aunque uno de los miembros de la familia percibe el subsidio por desempleo, la unidad familiar seguiría estando por debajo del umbral de pobreza: la posibilidad de acumular las dos prestaciones (RdC y NASPI, por ejemplo) mejoraría en cierto modo la situación de esa familia.

Es evidente que la cuantía percibida en concepto de prestación por desempleo contribuye a la hora de determinar los ingresos de las familias, resultando relevante para el acceso a la RdC y, por consiguiente, para la cuantía resultante.

Es también compatible con la RdC la percepción, por parte de uno o más miembros de la familia, de prestaciones asistenciales. Por regla general, también contribuyen a determinar los ingresos familiares pertinentes para el acceso a la RdC, a excepción de las "prestaciones no sujetas a comprobación de recursos", que podrán acumularse con la cuantía de la ayuda sin restricción alguna (párrafo 6 del artículo 2).

Como en el caso de NASPI y DIS-COLL, "no tendrá derecho a percibir la RdC, el miembro de la unidad familiar que se encuentre en situación de desempleo voluntario, durante los doce meses siguientes a la fecha del cese en el trabajo, excepto en el caso de cese por causa justificada" (art. 2, párrafo 3).

\section{5. - Consideraciones sobre los siguientes aspectos: b) los servi- cios de reinserción.}

Parlamento Europeo, Resolución de 20 de octubre de 2010 sobre el papel de la renta min en la lucha contra la pobreza y la promoción de una sociedad integradora en Europa, punto 15.

45. En base a las estimaciones realizadas por el Gobierno, cuando se presentó la política a los medios de prensa, se preveía que alrededor de 1,8 millones de personas se beneficiarían de la Rel, incluidos 700.000 menores (en comparación con los casi 5 millones de personas en situación de pobreza absoluta en 2017).

46. Se ha observado que "la distribución de los mayores fondos disponibles es, por lo tanto, injusta: la cantidad media de solteros aumenta en un $102 \%$ en comparación con la Rei, mientras que a medida que aumenta el número de familias, el aumento es más leve, hasta $+40 \%$ para los núcleos familiares de 5 y más miembros. No es de extrañar, pues, que los ingresos en concepto de ciudadanía sean superiores al umbral de pobreza absoluta para muchas familias de hasta 3 miembros (especialmente en el Sur, dado que se utiliza un único umbral nacional) y siempre inferiores para las que tienen por lo menos 4 miembros. Además, la Oficina Parlamentaria de Presupuesto informa que, en el caso de las personas solteras, la transferencia es superior a los valores medios de medidas similares en muchos países europeos, mientras que en el caso de las familias con dos o más hijos es inferior" (Gori 2019a). Toso 2019 expresa preocupaciones similares.
La naturaleza compuesta de la $\mathrm{RdC}$ requiere que la prestación ofrecida a las personas necesitadas no se limite al pago de un subsidio económico, sino que también continúe con la preparación de un proceso de reinserción a la vida laboral y/o social, destinado a superar y eliminar las condiciones subjetivas que han determinado la situación de pobreza. Los servicios de reintegración son, por lo tanto, el segundo pilar fundamental en el que se basa la arquitectura de la $\mathrm{RdC}$. Desde este punto de vista, la $\mathrm{RdC}$ reproduce, en sus líneas esenciales, el enfoque ya experimentado por la Rel, basado en la activación de los beneficiarios mediante la estipulación de "acuerdos" específicos: "el acuerdo para el empleo" y el "acuerdo para la inclusión social".

La conclusión del acuerdo constituye uno de los momentos en los que el carácter "condicional" del servicio es más evidente: a cambio del apoyo recibido, los miembros de 
las familias que reciben el subsidio deben asumir una serie de obligaciones, acordadas con los servicios competentes. En el párrafo 1 del artículo 4 se aclara que la concesión de la ayuda está sujeta a una doble condición: en primer lugar, los miembros mayores de edad de la familia deberán suscribir, a través de una plataforma digital especial, una "declaración de disponibilidad inmediata para trabajar" (denominada DID); en segundo lugar, los mismos obligados deberán asimismo adherirse a un "itinerario personalizado de acompañamiento para la integración laboral y la inclusión social que incluya la realización de actividades al servicio de la comunidad, la reconversión profesional, la finalización de estudios, así como otros compromisos identificados por los servicios competentes en vista a la integración al el mercado laboral y a la inclusión social”"47.

La decisión de recurrir a uno u otro tipo de acuerdo, depende de la evaluación de la situación de la familia en cuestión, con especial atención a las causas que han dado luga a la necesidad. Si el estado de pobreza se debe principalmente al desempleo/no ocupación de los miembros mayores de edad de la familia, el instrumento previsto para ello es el acuerdo para el empleo, destinado a la (re)integración rápida al mercado laboral. Si, po otra parte, se considera que la pobreza es "multidimensional"48, es decir, que se produce por un abanico más amplio de causas, se utilizará el acuerdo para la inclusión social, que prevé una serie más articulada y compleja de obligaciones de activación.

\section{1) El acuerdo para el trabajo}

El análisis de la noción de Renta Ciudadana, así como de las funciones que se le atribuyen, ha demostrado que la principal preocupación del legislador parece ser la de establecer una vía de reinserción laboral que permita a los beneficiarios del subsidio salir de la pobreza que los atrapa. Por eso, el instrumento legal propuesto para ello ha sido el Acuerdo para el Trabajo.

En principio, están obligadas a declarar su voluntad de trabajar, todas las personas mayores de 18 años desocupadas o que no estén estudiando. Sin embargo, se prevén ciertas excepciones de la regla:

- personas que reciben la pensión ciudadana o beneficiarios de la $\mathrm{RdC}$ que reciben una pensión directa o que tengan por lo menos 65 años de edad (como son personas mayores, no tiene sentido aspirar a su reinserción en el mercado de trabajo)

47. Alaimo 2020 subraya que el "camino personalizado hacia el empleo y a la inclusión social" es el marco general en el que se situarán el acuerdo para el trabajo y el acuerdo para la inclusión.

48. El enfoque multidimensional de la pobreza también es explícitamente acogido por la UE: véase Comisión Europea, La Plataforma Europea contra la Pobreza y la Exclusión Social: Un marco europeo para la cohesión social y territorial, $\operatorname{COM(2010)} 758$ final, Bruselas, 16.12.2010. personas discapacitadas, siempre que no les interese seguir las vías de integración laboral específicamente previstas para las personas discapacitadas en la Ley $\mathrm{N}^{\circ}$ $68 / 1999$;

- -los personas que asisten a los cursos de formación;

- las personas que tengan cargas familiares por cuidado de menores de tres años u otros miembros de la familia con discapacidad grave o que no sean autosuficientes.

En relación a estos sujetos, se observa una evidente atenuación del principio de condicionalidad: si asumimos como base de la condicionalidad el deber de realizar una actividad o función que contribuya al progreso material o espiritual de la sociedad - según lo dispuesto en el art. 4 , párrafo 2, Const. - ${ }^{49}$, el cumplimiento de este deber no sólo desemboca en el desempeño de una actividad laboral, sino también en otras actividades que, si bien no dan lugar a la manifestación de una "profesión" en sentido estricto, están dotadas de importancia social (por ejemplo, la labor de cuidado realizado en el seno del hogar) ${ }^{50}$

Las personas obligadas a suscribir la DID ("declaración de disponibilidad inmediata para trabajar") deberán suscribir asimismo el "acuerdo para el empleo"- un acuerdo mediante el cual se definen las obligaciones específicas de activación que deben cumplir los beneficiarios de la RdC, según lo acordado con los centros de empleo; de lo contrario se aplicarán sanciones (sobre este punto véase el párrafo siguiente) ${ }^{51}$.

Sin perjuicio de las actividades específicas que estipule el acuerdo, el párrafo 8, artículo 4. b), ofrece un ejemplo de contenido del acuerdo de activación que los beneficiarios deberán suscribir con el centro de empleo: "1) registrarse en la plataforma digital señalada [... y consultarla diariamente como apoyo a la búsqueda activa de empleo; 2) realizar una búsqueda activa de empleo, verificando la existencia de nuevas ofertas de trabajo, según las modalidades adicionales definidas en el acuerdo para el trabajo, que, sin embargo, se identificarán en el diario de las actividades que deben realizarse; 3) aceptar comenzar las actividades previstas en el acuerdo; 4) someterse a entrevistas de psico-aptitud y a

49. El artículo 4 de la Constitución italiana, después de reconocer el derecho al trabajo (párrafo 1), establece que "todo ciudadano tiene el deber de realizar, según sus posibilidades y su elección, una actividad o una función que contribuya al progreso material o espiritual de la sociedad": en esta disposición, la doctrina encuentra el fundamento del principio de la condicionalidad. Sobre la base constitucional del principio de condicionalidad, véase, por ejemplo, Armour 2013 y Ales 2015.

50. Ravelli 2018b, p. 598

51. El artículo 4,8.a), dispone que los beneficiarios de la RdC deberán cooperar con los centros de empleo en la definición del contenido del acuerdo para el trabajo. 
cualquier prueba de selección destinada a la contratación, según lo indicado por los servicios competentes y en relación a sus competencias; 5 ) aceptar al menos una de las tres ofertas de empleo acordes a sus competencias [...]"52

Además de las obligaciones previstas específicamente en el apartado b) del párrafo 8 del artículo 4, así como cualquier otra obligación que se determine en el acuerdo para el empleo (o para la inclusión social), se podrá exigir a los beneficiarios de la RdC que manifiesten su voluntad de trabajar en trabajos de utilidad pública, en el municipio de residencia ${ }^{53}$. La duración de la actividad, que en todo caso deberá ser compatible con e cumplimiento de los demás compromisos contraídos en virtud del acuerdo, oscila entre un mínimo de 8 y un máximo de 16 horas semanales (artículo 4.15).

\section{2) El acuerdo para la inclusión}

Como se ha visto, el acuerdo para el empleo es el instrumento de reintegración que se utilizará con carácter prioritario en todos los casos en que la pobreza de las familias se deba principalmente al desempleo o desocupación. Sin embargo, en muchos casos, la pobreza está determinada no sólo por la falta de trabajo -que es una de las causas más importantes, sino también por otros factores que, al interactuar con el desempleo, hacen que la situación de las familias sea particularmente gravosa.

Cuando la pobreza adquiere el carácter de "multidimensionalidad", los beneficiarios de la RdC están invitados a firmar un acuerdo más complejo y articulado, en el que participan, además de los centros de empleo, los servicios sociales competentes: se trata de llamado "acuerdo para la inclusión social" cuya disciplina nos recuerda, por expresa refe-

52. La idoneidad, tanto para los amortiguadores sociales como para la RdC, se mide en función de tres parámetros principales: a) la coherencia entre la oferta de empleo y la experiencia y las competencias adquiridas; b) la distancia del lugar de trabajo al domicilio y los tiempos de traslado en transporte público; c) la duración del estado de desempleo. Los parámetros generales para la adecuación de la oferta de trabajo se rigen por el Decreto Ministerial de 10 de abril de 2018. Sin perjuicio de la aplicación de estos parámetros, la reglamentación de la RdC - véase el párrafo 9 de artículo 4 - introduce una modulación diferente de los requisitos de idoneidad relacionados con la distancia, actuando como lex specialis

53. Estas actividades de utilidad pública - que se llevarán a cabo en el ámbito cultural, social, artístico, ambiental, de formación y de protección del bien común - deben, en principio, ser coherentes con las responsabilidades del individuo obligado. Las actividades se establecen en base a los proyectos gestionados por los municipios. rencia legislativa, al llamado "proyecto personalizado" previsto por el Decreto Legislativo ${ }^{0} 147 / 2017$ sobre la R.e. ${ }^{54}$

A diferencia del otro, el acuerdo para la inclusión social queda abierto a otros contenidos: el objeto del acuerdo no son sólo las actividades destinadas a la reinserción laboral, que en cualquier caso siguen representando el núcleo principal del compromiso asumido por los beneficiarios, sino también una gama más amplia de conductas, que pueden ncluir la "asistencia a la escuela" (para los menores) y, si es necesario, "conductas de prevención y tratamiento destinadas a la protección de la salud"55. El procedimiento que conduce a la conclusión del acuerdo consta de tres fase ${ }^{56}$. La primera prevé la realización de un "análisis preliminar" destinado a identificar las

necesidades de todo el núcleo familiar y sus componentes individuales. Este es un primer perfil que sirve para guiar los siguientes pasos del procedimiento. Si el elemento de vulnerabilidad del núcleo familiar se limita únicamente a la falta de trabajo, el instrumento adecuado es el acuerdo para el empleo. En caso de que surjan nuevos perfiles de vulnerabilidad como resultado del análisis preliminar, se lleva a cabo una evaluación más profunda, denominada "cuadro de análisis". Este segundo paso es necesario para la "construcción de la evaluación multidimensional de las unidades familiares con necesidades complejas", posteriores y diferentes de la simple falta de empleo. La definición del cuadro de análisis se confía a un "equipo multidisciplinario" integrado por expertos provenientes de los servicios sociales y de salud, identificados en base a las necesidades específicas detectadas durante el análisis preliminar.

54. Para un primer comentario ver Mandrone, D’Emilione 2019. La justificación del acuerdo para la inclusión se aclara en las directrices para la definición de los acuerdos para la inclusión socia emanadas del Ministerio de Trabajo y Política Social: "la justificación del Acuerdo para la Inclusión Social parte de que los ingresos por sí solos no bastan para salir de la pobreza, ya que la falta de ingresos no suele ser la causa de la pobreza, sino su efecto. Las causas pueden ser diferentes y típicamente multidimensionales. Para evitar las "trampas de la pobreza" es importante actuar sobre as causas con un diseño personalizado que identifique las necesidades y los recursos de cada hogar, prepare las intervenciones adecuadas y lo acompañe hacia la autonomía. Es un camino en e que los servicios en red -servicios sociales, sanitarios y sociales y centros de empleo, con carácter prioritario- asumen una clara responsabilidad hacia los ciudadanos más vulnerables y se comprometen -"se activan"- en el marco de un Pacto personalizado acordado conjuntamente" (pág. 13)

55. Véase el artículo 6, párrafo 5, letra d), del Decreto Legislativo $N^{\circ} 147 / 2017$, al que se refiere expresamente la Ley $N^{\circ} 26 / 2019$

56. Las tres fases se describen detalladamente en las Directrices para la definición de los acuerdos para la inclusión social del Ministerio de Trabajo y Política Social. 
La tercera fase consiste en una verdadera "planificación personalizada", en la que se define el contenido del acuerdo para la inclusión, especificando la conducta activa que se requiere a los beneficiarios de la $\mathrm{RdC}$.

En cuanto al contenido del acuerdo, la Ley $n^{\circ} 26 / 2019$ no proporciona ninguna pauta en particular, remitiéndose expresamente a la disciplina del "proyecto personalizado" a la que se refiere el artículo 6 del Decreto Legislativo $n^{\circ}$ 147/2017 sobre la Rel.

La identificación de los contenidos del proyecto personalizado prevé que se establezca un proceso de puesta en común de objetivos, instrumentos y resultados esperados entre la familia en cuestión y los servicios competentes: estos elementos se someten a una suerte de "negociación con los beneficiarios, con la recomendación de evitar expresiones técnicas, genéricas o abstractas" ${ }^{27}$.

El método elegido, basado en la participación de los beneficiarios y los entes públicos en un procedimiento de negociación, tiene por objeto alcanzar el mejor equilibrio posible entre los intereses públicos y privados implicados. Por consiguiente, se promueven métodos para definir el contenido y la duración del proceso que respeten los principios de "proporcionalidad, idoneidad y no exceso, en relación con las necesidades de apoyo de grupo en cuestión" 58 .

El cumplimiento del principio de proporcionalidad tiene por objeto, como ya se ha dicho, salvaguardar los intereses de los dos sujetos implicados. El primer interés, especificado por el legislador, es referente a la parte pública y consiste en la necesidad de que la acción administrativa se lleve a cabo en el marco de la economía de medios de forma coherente con los recursos disponibles - actualmente bastante limitados - y su correcta asignación. El segundo interés, implícito pero no menos relevante, se re fiere a los beneficiarios de la ayuda, que, a cambio de las medidas de apoyo, deben asumir un conjunto de obligaciones que en algunos casos pueden invadir la intimidad de aquellos: existe un riesgo elevado de que el apoyo recibido, gracias al poderoso efecto palanca de la condicionalidad, desemboque en una compresión a la libertad de autodeterminación de las personas. Por lo tanto, sería adecuado que esta compresión, si es que va a existir, no exceda de lo estrictamente necesario para lograr los objetivos de la inclusión ${ }^{59}$

57. art. 6 , comma 3 , lett. b), d.lgs. n. 147/2017.

58. art. 6, comma 7, d.Igs. n. 147/2017.

59. Ravelli 2018a, pp. 177-178.

\section{La condicionalidad y el sistema sancionador.}

La adhesión a la "vía personalizada de apoyo a la inserción y a la inclusión social" y el cumplimiento de las obligaciones del acuerdo constituyen una condición para el acceso a la prestación y su mantenimiento a lo largo del tiempo ${ }^{60}$.

El enfoque de la cuestión de condicionalidad adoptado por el legislador en los últimos años, especialmente desde la Reforma Fornero de 2012, va en consonancia con la tendencia que se ha afirmado progresivamente en Europa, especialmente en lo que respecta a la flexiseguridad: el modelo de integración entre las políticas activas y pasivas que predomina actualmente en nuestro continente ha dado lugar a la puesta en marcha de "mecanismos coercitivos basados en el sistema carrot and stick, según el cual la percepción monetaria de la prestación y la participación en las iniciativas de reinserción al mercado laboral, están sujetas a obligaciones cada vez más estrictas para los perceptores." ${ }^{1}$

Esta estrategia sólo puede apoyarse en un aparato sancionador adecuado, que sea capaz de emitir una fuerza persuasiva suficiente para orientar la conducta de los beneficiarios de la prestación, orientándola hacia modelos de comportamiento considerados "virtuosos" y acordes a los objetivos de la ley, perseguidos por el Legislador

El incumplimiento de una o más de las obligaciones asumidas en virtud del acuerdo, desencadenaría en una reacción que se traduciría en la aplicación de una serie de sanciones caracterizadas por un grado de afectación proporcional al número y a la gravedad de las infracciones.

Un primer grupo de conductas -menos graves- prevé la aplicación de sanciones atendiendo al principio de gradualidad (sanciones que van desde la reducción del subsidio hasta su confiscación en caso de reincidencia). La sanción se aplicaría en caso de: a) no acudir a las llamadas realizadas por los centros de empleo y destinadas a evaluar las necesidades de la unidad familiar; b) no participar en las iniciativas de orientación para la reinserción laboral; c) no cumplir con los compromisos previstos en el acuerdo para la

60. Las medidas de condicionalidad previstas por el legislador que se impondrán a los perceptores de la RdC, se han suspendido temporalmente en virtud del artículo 40 del decreto legislativo № 18, de 17 de marzo de 2020 (el denominado "Cura Italia"), a fin de limitar al máximo los viajes, habida cuenta de la emergencia provocada por el Covid-19. La duración de la suspensión, fijada inicialmente en dos meses, fue prorrogada por otros dos meses en virtud del artículo 76 del Decreto Legislativo № 34 de 19 de mayo de 2010 (el denominado "Decreto de relanzamiento"). Sin embargo, es una suspensión que encuentra su razón de ser en la situación de emergencia: por lo tanto, debe considerarse como una intervención totalmente contingente, que no cambia la naturaleza -condicional- de la RdC.

61. Caruso, Cuttone 2015, p. 12 
inclusión, relativos a la asistencia a cursos de educación/formación de los miembros menores de edad, o a los cursos sobre "conductas de prevención y tratamiento destinadas a la protección de la salud". Ello se entiende sin perjuicio del caso en que el incumplimiento se deba a una razón justificada ${ }^{62}$

Un segundo grupo de conductas, evaluadas con mayor severidad, implica la pérdida inmediata del beneficio. Estos serían los casos en los que uno o más miembros de la familia ${ }^{63}$ :

- se niega a realizar las actividades de reinserción a las que el legislador atribuye un valor particular como, por ejemplo la DID, la firma del acuerdo, la participación en iniciativas de readaptación profesional, la participación en el programa de actividades de utilidad social (preparado por el municipio de residencia);

- realiza declaraciones falsas para obtener la prestación (o para obtener una prestación superior a la cantidad que le corresponda) o no realiza las declaraciones obligatorias (por ejemplo, las relativas a cambios en los ingresos y/o activos financieros);

- rechaza tres ofertas de trabajo o, en caso de renovación de la $\mathrm{RdC}$, rechaza la primera oferta de trabajo correspondiente a sus competencias;

- se encuentra, tras una inspección, realizando trabajos sin haberlo comunicado a los servicios competentes.

Algunas de las conductas más graves (por ejemplo, las declaraciones falsas) también se castigan mediante instrumentos promovidos por el derecho penal.

Cabe señalar que las sanciones funcionan incluso cuando los incumplimientos son atribuibles a uno solo de los miembros de la familia: el incumplimiento de uno de ello produce efectos sobre todos los demás. Se trata de una medida bastante drástica, que refleja una concepción subyacente del núcleo familiar como un organismo unitario y no como un grupo compuesto por individuos independientes, que también pueden tener relaciones conflictivas con el resto del grupo, que no siempre se dirigen fácilmente hacia objetivos compartidos (este tipo de conflictos dentro de las familias no es poco frecuente especialmente en contextos particularmente desfavorecidos). La responsabilidad individual tiende así a transformarse en una responsabilidad colectiva, solidaria, en virtud de la cual los efectos perjudiciales derivados del fracaso del individuo se transmiten a los demás componentes: se crea una especie de presunción de que la culpa del individuo es atribuible a todo el grupo y por lo tanto se castiga como tal. Parece ser una interpretación algo arcaica de la responsabilidad, pero se ve afectada por la decisión de construir la la renta de inserción sobre una base familiar, más que individual.

62. art. 7, commi 7-8-9

63. art. 7 , commi $5-6$.

\section{Observaciones finales}

El hecho de que el ingreso mínimo se introdujera en Italia hace poco más de dos años y de que se haya producido un importante cambio legislativo in itinere, dificulta la elaboración de un balance preciso y fiable. Sin embargo, es posible hacer algunas observaciones provisionales, destacando algunos perfiles críticos que surgieron durante la primera fase de aplicación.

Es indudablemente algo positivo el hecho de que el número de beneficiarios de la ayuda haya aumentado de 1,8 millones a unos 5 millones. Desde este punto de vista, el legislador ha demostrado que se ha tomado en serio una de las principales críticas formuladas por la doctrina referida a la Rel, en concreto, que se trataba de una medida de tamaño insuficiente.

Dicho esto, es precisamente la ampliación del número de beneficiarios lo que debería considerarse una mejora importante de esta política, pero que a la vez, plantea una serie de problemas que no son fáciles de resolver. La gestión de un número casi triplicado de posibles beneficiarios y la necesidad de contener el riesgo de conductas oportunistas ha levado al legislador a introducir una política más compleja y articulada, que en muchos casos ha resultado difícil de aplicar por aquellas instituciones que aún no están preparadas para hacer frente a un reto tan exigente.

Además de ejercer presión sobre las oficinas públicas encargadas de prestar los servicios relacionados con la $\mathrm{RdC}$, la complejidad de la medida ha sido un obstáculo para muchas personas pobres que, para no tener que enfrentarse a un procedimiento engorroso y a veces invasivo de la esfera personal de los miembros de la familia, han preferido no solicitar el acceso a la misma. Ello plantea graves problemas de aceptación por parte de la población, tal como lo demuestran los datos sobre el número de solicitudes presentadas y el número total de personas involucradas ${ }^{64}$ : el miedo a ser excluidas de la prestación viene precisamente de las familias más frágiles y menos preparadas para hacer frente a situaciones de necesidad 65 .

64. Los datos se encuentran en la página web del Observatorio del INPS sobre Ingresos de la Ciudadanía: https://www.inps.it/webidentity/banchedatistatistiche/redcit/index.jsp. Al 31 de mayo de 2019, seis meses después de la introducción de la RdC, habían sido admitidas unas 674 mil solicitudes y rechazadas, 277 mil. La situación ha mejorado en 2020. El INPS ha publicado los datos correspondientes al mes de febrero, que muestran la siguiente situación: alrededor de 871 mil hogares se beneficiaron la RdC, un total 2 millones 321 mil personas; alrededor de 118 mil familias reciben la $\mathrm{PdC}$, un total de 134 mil personas. Sin embargo, en comparación con el número de personas que viven por debajo de umbral de pobreza, la medida no ha alcanzado todavía plenamente su objetivo. Para obtener datos más detallados, visite: https://www.inps.it/webidentity/banchedatistatistiche/redcit/index.jsp.

65. Gori 2019b. 
También hay un tercer aspecto crítico, que se refiere a la limitada capacidad institucional de los centros de empleo encargados de gestionar la delicada fase de reintegración (o primera colocación en el empleo) de los beneficiarios de la RdC. Se trata de un punto muy delicado, sobre todo si se considera que la RdC pretende ser, incluso antes de ser un instrumento de lucha contra la pobreza y la exclusión social, "una medida fundamental de política laboral activa que garantice el derecho al trabajo" (art.1.1). El buen funcionamiento de los instrumentos de la política activa de empleo es, pues, esencial para la consecución de los objetivos generales que ha fijado el legislador; Por desgracia, son precisamente los centros de empleo, que deben ser el eje en torno al cual giren las vías de reinserción laboral, los que constituyen hoy el principal punto débil de la arquitectura diseñada por la Ley № 26/2019.

El Gobierno ha planteado la cuestión del fortalecimiento de los centros de empleo ${ }^{66}$ pero los resultados obtenido, a día de hoy, no se muestran satisfactorios.

66. Por ejemplo, en 2019, se contrataron 3.000 figuras profesionales - los llamados "navegantes" - para acompañar al beneficiario de la RdC en el difícil camino de la reintegración. Sin embargo, estas figuras profesionales aún no han sido formadas y, por lo tanto, no podrán, al menos durante un tiempo, llevar a cabo con eficacia las tareas que se les asignen. Además, y esto es una verdadera paradoja, los navegantes son contratados con un contrato de colaboración de duración determinada, por lo que ellos mismos trabajan en situaciones de precariedad laboral, y serán destinados -a corto plazo- a enfrentarse al problema del desempleo. 


\section{Referencias bibliográficas}

Alaimo A. (2017), II reddito di inclusione attiva. Note critiche sull'attuazione della legge $n$ 33/2017, in Rivista del diritto della sicurezza sociale, n. 3, p. 419 ss.

Alaimo A. (2019), Il reddito di cittadinanza fra diritto all'assistenza e doveri di attivazione. Per un modello idealtipico di strategia di inclusione, in Variazioni su Temi di Diritto de Lavoro, n. 2, p. 457 ss.

Alaimo A. (2020), Il reddito di cittadinanza: il beneficio economico, il patto per il lavoro e il patto per l'inclusione sociale, in Giubboni S. (a cura di) (2020), Reddito di cittadinanza e pensioni: il riordino del welfare italiano, Torino, Giappichelli (in corso di pubblicazione)

Ales E. (2015), Diritti sociali e discrezionalità del legislatore nell'ordinamento multilivello: una prospettazione giuslavoristica, in Giornale di diritto del lavoro e di relazioni industriali, n. 3, p. 455 ss.

Apostoli A. (2016), Il consolidamento della democrazia attraverso la promozione della solidarietà sociale all'interno della comunità, in Costituzionalismo.it, 1, p. 35 ss.

Balandi G.G. (1987), voce Assicurazione sociale, in Digesto discipline privatistiche, sez. comm., Torino, Utet, p. 379 ss.

Baldini M., Gori C. (2019), II reddito di cittadinanza, in II Mulino, n. 2, p. 269 ss.

Bozzao P. (2018), Poveri lavoratori, nuovi bisogni e modelli universalistici di welfare: qual tutele?, in Lavoro e diritto, n. 4, p. 657 ss.

Bronzini G. (2011), Il reddito minimo garantito nell'Unione europea: dalla Carta di Nizza alle politiche di attuazione, in Giornale di diritto del lavoro e di relazioni industriali, $\mathrm{n}$. 2, p. 225 ss.

Caruso B., Cuttone M. (2015), Verso il diritto del lavoro della responsabilità: il contratto di ricollocazione tra Europa, Stato e Regioni, WP CSDLE "Massimo D'Antona".IT 283/2015, p. 1 ss.

Casillo R. (2019), Il reddito di cittadinanza nel d.I. 28 gennaio 2019, n. 4: precedenti, luci, ombre, in Rivista del diritto della sicurezza sociale, 3, p. 557 ss.

Corazza L. (2013), II principio di condizionalità (al tempo della crisi), in Giornale di diritto del lavoro e di relazioni industriali, n. 3, p. 489 ss.

D’Onghia M. (2020), Il Reddito di Cittadinanza un anno dopo: eppur si muove ma con troppe funzioni e a doppia velocità, in Labor - Il lavoro nel diritto, n. 1, 2020, p. 27 ss.
Fellini I. (2015), Una «via bassa» alla decrescita dell'occupazione: il mercato del lavoro italiano tra crisi e debolezze strutturali, in Stato e mercato, p. 469 ss.

Giubboni S. (a cura di) (2020), Reddito di cittadinanza e pensioni: il riordino del welfare italiano, Torino, Giappichelli (in corso di pubblicazione)

Gori C. (2019a), Reddito di cittadinanza: il punto diopo il decreto, in lavoce.info, 5 aprile 2019, testo reperibile al sito: https://www.lavoce.info/archives/58400/reddito-di-cittadinanza-il-punto-dopo-il-decreto/

Gori C. (2019b), Ma tanti poveri non chiedono il RdC. Ecco perché, in lavoce.info, 18 giugno 2019, testo reperibile al sito: https://www.lavoce.info/archives/59724/redditodi-cittadinanza-il-nodo-di-chi-non-presenta-la-domanda/

Granaglia E., Bolzoni M. (2016), II reddito di base, Roma, Ediesse

Loy G. (2009), Una Repubblica fondata sul lavoro, in Giornale di diritto del lavoro e relazioni industriali, n. 2, p. 197 ss.

Mandrone E. D’Emilione M. (2019), Il patto per l'inclusione: la funzione sociale del reddito di cittadinanza, in Marocco M., Spattini S. (2019), Diritto al lavoro, contrasto alla povertà, politica attiva, inclusione sociale: le tante (troppe?) funzioni del reddito di cittadinanza all'italiana Primo commento al d.I n. 4/2019, ADAPT Labour Studies e-Book series, n. 79, p. 53 ss

Nogler L. (2009), Cosa significa che l'Italia è una Repubblica "fondata sul lavoro"?, in Lavoro e diritto, n. 3, p. 427 ss.

Pedrazzoli M. (2011), Assiologia del lavoro e fondamento della Repubblica: il lavoro è una "formazione sociale"?, in Quaderni cost., n. 4, p. 969 ss.

Peña-Casas R., Ghailani D., Spasova S., Vanhercke B. (2019), In-Work Poverty in Europe. A Study of National Policies, European Social Policy Network, Brussels: European Commissione

Raitano M., Jessoula M., Natili M., Pavolini E. (2019), I working poor in Italia, in Social Cohesion Papers, n. 2, p. 1 ss.

Ravelli F. (2018a), Il reddito minimo tra universalismo e selettività delle tutele, Torino, Giappichelli

Ravelli F. (2018b), Condizionalità e "merito" nel sistema di sicurezza sociale italiano, in Diritti, lavori, mercati, n. 3, p. 585 ss.

Ravelli F. (2019), L'accesso alle prestazioni di previdenza e di assistenza: conta ancora la cittadinanza?, in Calore A., Mazzetti F. (a cura di), I confini mobili della cittadinanza, Torino, Giappichelli, p. 167 ss. 
Ravelli F. (2020), Natura e inquadramento costituzionale del reddito di cittadinanza, in Giubboni S. (a cura di), Reddito di cittadinanza e pensioni: il riordino del welfare italiano, Torino, Giappichelli, p. 15 ss. (in corso di pubblicazione)

Reyneri E. (2015), Anti-poverty policies and the (decreasing) social cohesion in

Europe. A comment, in Stato e mercato, p. 97 ss.

Rosafalco A. (2019), Reddito di cittadinanza e cittadini extra-europei, in Marocco M., Spattini S. (2019), Diritto al lavoro, contrasto alla povertà, politica attiva, inclusione sociale: le tante (troppe?) funzioni del reddito di cittadinanza all'italiana Primo commento al d.I n. 4/2019, ADAPT Labour Studies e-Book series, n. 79, p. 81 ss.

Saraceno C. (2015), Il lavoro non basta. La povertà in Europa negli anni della crisi, Milano, Feltrinelli

Sigillò Massara G. (2019), Dall'assistenza al Reddito di Cittadinanza (e ritorno). Prime riflessioni sul Reddito di Cittadinanza, Torino, Giappichelli

Toso S. (2016), Reddito di cittadinanza. O reddito minimo?, Bologna, II Mulino

Toso S. (2019), E ora la parola ai fatti. Pregi e difetti del reddito di cittadinanza, con qualche suggerimento per il futuro, 8 aprile 2019, testo reperibile al sito: https://welforum. it/e-ora-la-parola-ai-fatti/

Van Parijs P. (1991), Why Surfers Should be Fed: The Liberal Case for an Unconditional Basic Income, in Philosophy and Public Affairs, 20:2, p. 101 ss.

Vincieri M. (2017), Verso la tutela della povertà: l'ipotesi del reddito di inclusione, in Lavoro e diritto, n. 2, p. 301 ss.

Vincieri M. (2020), Spunti critici sul reddito di cittadinanza, in Diritto delle relazioni industriali, n. ??? (in corso di pubblicazione) 